\title{
Téoros
}

Revue de recherche en tourisme

\section{Rôle et action des associations touristiques régionales}

\section{Normand Cazelais}

Volume 5, numéro 1, mars 1986

Tourisme et régions

URI : https://id.erudit.org/iderudit/1080593ar

DOI : https://doi.org/10.7202/1080593ar

Aller au sommaire du numéro

Éditeur(s)

Université du Québec à Montréal

ISSN

0712-8657 (imprimé)

1923-2705 (numérique)

Découvrir la revue

Citer cet article

Cazelais, N. (1986). Rôle et action des associations touristiques régionales.

Téoros, 5(1), 30-32. https://doi.org/10.7202/1080593ar d'utilisation que vous pouvez consulter en ligne.

https://apropos.erudit.org/fr/usagers/politique-dutilisation/ 


\section{Rôle et action des associations touristiques régionales}

par Normand Cazelais *

Les Associations touristiques régionales -Ou ATR-sont des acteurs de premier plan au sein des forces vives qui animent le développement et l'aménagement régional au Québec. Leur action s'exerce selon deux axes fondamentaux. Le premier est verti= cal et sectoriel: les ATR regroupent des intervenants très divers, depuis les centres de ski et les hôteliers jusqu'aux municipalités et theàtres d'été, autour d'un méme centre d'intérét. le tourisme. Le second est. a la fois spatial et horizontal: par définition, leur champ d'intervention privilégié est la région, entité plus ou moins homogène qui constitue le tissu nerveux de tout territoire national organisé. Certes, les ATR n'ont pas pour mandat ni róle de coordonner le développement et l"aménagement régional, mais de participer intimement à la définition du produit touristique local et régional, de susciter sa mise en valeur et d'assurer sa promotion et sa diffusion selon divers horizons. Par cette double action, les ATR -qui comptèrent parmi les premiers représentants de la décentralisation et de la régionalisation- contribuent-elles, de façon directe et significative à l'évolution de ce cadre plus vaste défini par le développement et l'aménagement régional.

\section{Une première enquête}

Aussi apparut-il essentiel, dans l'approche et I'analyse $d$ 'un tel thème, $d$ 'aller chercher l'opinion de ces organismes qui oeuvrent sur le terrain, de ces acteurs "immediats" pourrait-on dire. Recueillir leurs commentaires sur cette question au terme de leur première septaine -el donc au sein de ce que les educateurs appelaient l'àge de raison..., est d'autant plus important que les ATR ont pu se consolider au cours de ces années. établir des rapports particuliers avec les différents secteurs de l'activité touristique d'une part, et avec les municipalités, ministeres et autres organismes qu façonnent le développement et $\mathrm{I}^{+}$aménagement touristiques d'autre part. D'autant plus important que sont apparues depuis lors les municipalités régionales de comté

"Normand Cazalais est chroniqueur en tourisme as charge de cours au departement de geographie de I'UOAM.
(MRC) qui parachèvent présentement leurs schémas d'aménagement alors que les ATR elles-mëmes se sont engagées dans l'élaboration de leurs propres plans de développement touristique régional.

Cette rapide enquête entrainera, j'en suis persuadé, de multiples retombées. Outre la production de ce texte qui n'est qu'une première analyse des réponses obtenues, elle en alimentera, n'en doutons pas, une étude plus approfondie qui devrait donner lieu par la suite à d'autres textes et articles s'attachant à des aspects plus spécifiques ou encore à une décortication plus poussée de la modulation des réponses en fonction de leurs origines géngraphiques. Elle devrait également susciter, au cours des mois à venir, si l'on se fie du moins aux commentaires verbaux qui nous ont été fajts. un ensemble de questions et de réflexions autour de ce theme.

La méthode d'enquête utilisée, compte tenu du court laps de temps alloué et de la portée exploratoire tant de ce numéro de Téoros que du texte lui-méme, fut celle d'un questionnaire ouvert, laissant libre cours aux répondants de formuler leurs opinions comme ils l'entendaient. Les questions. peu nombreuses, furent conçues pour donner un aperçu à la fois rétrospectif et prospectif de la place du tourisme dans le developpement et l'aménagement régional et pour y cerner le contexte et le rôle des ATR.

Compte tenu du fait que le questionnaire fut posté en hiver -à la veille des Fétes!et qu'en cette saison certaines ATR des régions les plus éloignées ne peuvent alors disposer d'un personnel permanent. le taux et la représentativité des réponses sont plus que satisfaisants. En effet, douze ATR (sur 18) ont répondu: Bas-Saint-Laurent, Abitibi-Témiscamingue, Saguenay-LacSaint-Jean-Chibougamau. Gaspésie. Estrie. Coeur-du-Québec. Pays-de-l'Erable. Montérégie, Grand-Montréal, Laurentides, De Lanaudière et Charlevoix.

La méthode de dépouillement fut la sujvante: dégager pour chacune des questions les grandes lignes de force et les constantes, meture en relief les principales nuan-

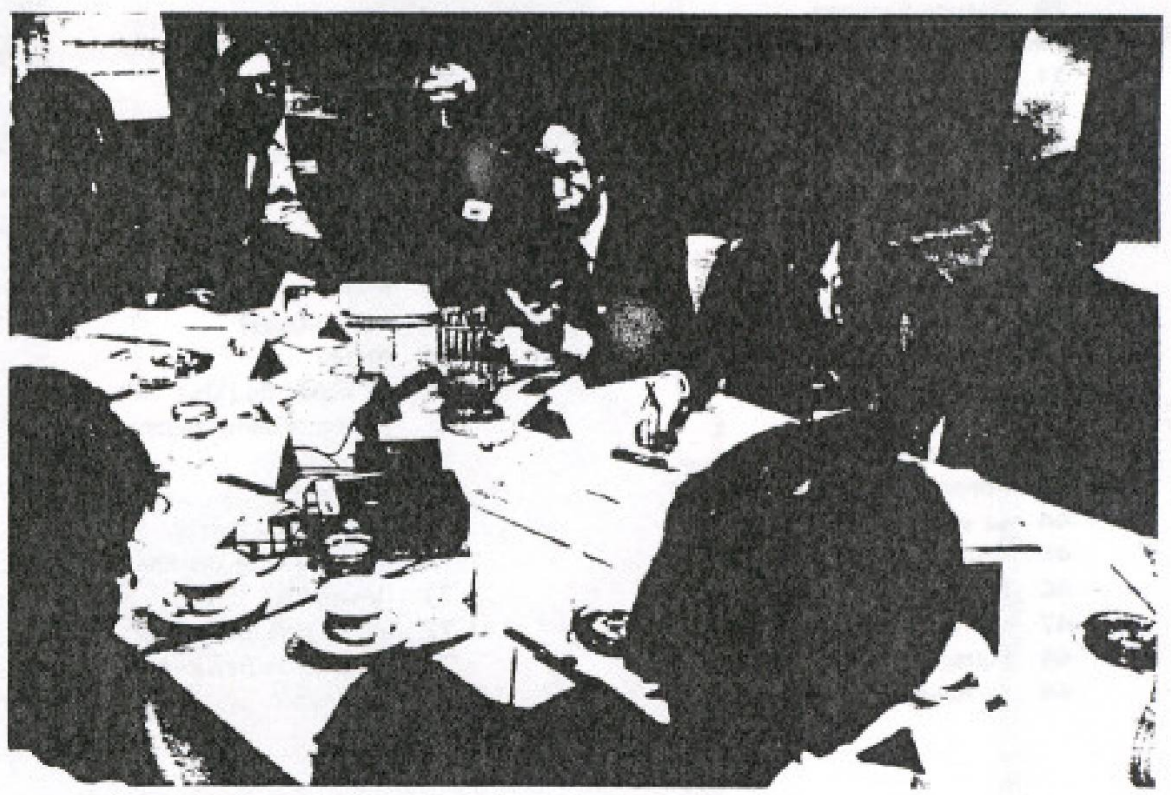

II Y a deux ans déjä, lors de la Journèe mondiale du tourisme -septembre 1984" un atelier était consacré au bilan des réalisations touristicues au niveau des MRC. Plusieurs représentants d"ATR y participaient. 
ces en identifiant leurs auteurs et citer. quand il y avait lieu de le faire, les commentaires les plus représentatifs ou les plus percutants.

"Quels sont, à votre avis, le rôle et I'importance du tourisme dans le développement et l'aménagement régional?"

Toutes les ATR s "entendent pour conférer au tourisme une grande importance en ce domaine "vital" (Grand-Montreal). On invoque, pour ce faire, son impact sur la création d'emplois, ses retombées économiques, la diversification qu'il apporte à l'économie tant québecoise que régionale, l'amélioration des conditions de vie qu'il procure. Cette "industrie" ferait partie du "virage technologique" (Estrie).

De façon plus spécifique, le tourisme constitue une "ressource renouvelable" (Abitibi-Témiscamingue) et une "altemative à un développement trop souvent axé sur les marières premieres d'une region " (Saguenay-Lac-Saint-Jean-Chibougamau). Appelé a supporter "'une partie croissante de l'économie" (Lanaudière), il est " $r$ " butaire de toutes les actions des hommes. que ce soit pour les infrastructures ou la qualité de la vie" (Charlevoix). Le tourisme permet également "la mise en valeur $d$ 'attraits naturels, culturels et historiques et peut contribuer à la sauvegarde de certains elements de notre patrimoine. $L$ accès à ces divers sites constitue par la suite un véhicule d'information privilegié, un ouril de diffusion" "(Bas-Saint-Laurent).

'Bien planifié, l'aménagement touristique permet une harmonisation des facteurs économiques, sociaux el géographigues d'une région" (Pays-de-1'Ërable). "A cause de sa complexité méme, le tourisme peut jouer un role important dans le développement régional er dans celui de nombrew secteurs d'activités comme le transport, l'hébergement, la restauration, les divertissements, les ans. Il peut ainsi contribuer à anténuer les disparités régionales" (Laurentides). Mais, souligne cette dernière ATR, "l importance du tourisme d ce chapitre warie beaucoup d'une région à l'autre: toutes $n$ 'ont pas les ressources nécessaires, les attraits et le potentiel pour faire du tourisme une activité importante".

\section{Rôle et importance du tou- risme dans votre région}

Malgré la nuance apportée par l'ATR des Laurentides, toutes les ATR accordent au tourisme beaucoup d'importance dans le développement et l'aménagement de leurs régions respectives, sans toutefois toujours donner la première place au sein des activités économiques; il tient un "rôle secondaire, de support et d'appui" (Coeur-du-Québec) ou encore celui d'une "industrie de transition entre le déclin des secteurs mous et la venue d'industries de pointe" (Estrie).

Plusieurs ATR identifient le besoin de réenligner la définition et le développement de leur produit touristique et voient dans le Plan de développement touristique régional l'occasion de le faire et aussi de s'insérer plus adéquatement au sein du développement et de l'aménagement régio. nal. Exemple: "La situation de notre région à proximité du grand bassin métropolitain et les pressions croissantes de ces visiteurs commandent une préoccupation en matière de développement el d amenage. ment régional afin de préserver le miliew d'accueil et de maximiser les retombees économiques" "(Lanaudière)

Deux opinions retiennent plus particulièrement l'attention qui, pour être différentes, $n$ 'en contiennent pas moins en filigrane des convergences et des préoccupations semblables de prises en mains autonomes. D'une part, I'influence de Silicone Valley: " $L$ 'ATE doir favoriser une qualité de vie essentielle à la mise en place d'un complexe culturo-récréo-touristique régional de classe, aboutissant d une relance indusrielle ordonnée de l'Estrie, notamment dans le secteur de pointe: l'ére des axes touristico-industriels" (Estrie). D'autre part, une certaine exaspération: "En Gaspésie, nous avons etté, depuis le $B A E Q$. aménagés et déménagés, et ceci ne s'est pas amélioré avec le Choix des regions. Que dire devant toutes ces manifestations politiques qui souvent, sous prétexte de permettre aux Gaspésiens de rantraper leur retard économique, ont détruit le sens mème de leur existence, de son cours évolutif normal et traditionnel. Ici, qui parle d'amenagement parle surtout de developpement, donc de Pouvoir. Comme vous le savez sans doute, le Bas-Saini-Laurent et la Gaspésie ont été et servent encore de laboratoires, ce qui n'ameliore pas nécessairement notre sort' (Gaspésie). Et l'auteur de suggérer aux intéressés la lecture d'un ouvrage paru récemment aux Éditions Asticou, sous la signature d'Alain G. Gagnon, Développement régional, Etat et Groupes populaires.

\section{Quelles sont les possibilités et limites des ATR dans cette action notamment dans leurs relations avec le ministère du Tourisme, les autres ministè- res, les MRC, etc?}

De l'avis général, les ATR ont un rôle de leader en matière de tourisme. Mais elles " $n$ 'ont pas les ressources financières pour intervenir directement dans le developpement" "Abitibi-Témiscamingue), comme promoteurs. Leur mandat, tel que stipulé dans le protocole d'entente qu'elles ont toutes signé avec le ministère du Tourisme du Québec -dont elles sont en région les "interlocuteurs privilégiếs" - est d"assurer "la promotion el la concertation touristiques régionales" (Charlevoix). Malgré cette reconnaissance, une ATR n'aurait 'en région qu un poids relarifen raison de sa structure juridique et administrative (corporation à but non lacratif)" (Bas-SaintLaurenty. Cependant, "un appui solide du ministère du Tourisme aux ATR esil le gage de l'aménagement plus harmonieux d'une région touristique et d'une planificarion greffé à la réalite" (Estrie).

"Le rôle de developpeur des ATR est encore embryonnaire car le partage des responsabilités à ce niveau entre les ministères manque de definision. Ainsi, IOPDQ. le $M L C P$, par exemple, disposent de programmes d'investissement directs, alors que le ministère du Tourisme ne possède pas de programme pour les ATR afin dintervenir dans le développement d'infrastructures" (Pays-de-1'Érable). Toutefois, les ATR font état de leur récent engagement dans l'élaboration, de leurs Plans de développement touristique régional respectifs, en collaboration avec le ministère du Tourisme qui assure une contribution financière.

Ce Plan est considéré comme un outil de travail qui sera fort précieux. Il "sera exécuté en consultation avec les divers schemas damenagement des $M R C$ de la région " (Montérégie). II "devra s'alimenter aux différentes érudes déjà réalisées dans le cadre de ces schémas; il sera un cadre de reference commun pour tous les intervenants touristiques prives et publics et pemettra de guider leurs actions et interventions vers un développement touristique cohérent" (Laurentides). Et, précise-t-on, "Dans la mesure aù le Plan de développement sera largement accepté par l'ensem= ble des intervenants concernes, s'etabliront des consensus sur des projets de développement rouristique" (Abitibi-Témiscamingue).

Dans 1'ensemble, les relations avec les municipalités sont assez bonnes: nombre de celles-ci sont membres d'ATR et, depuis le temps, ATR et municipalités ont appris à vivre les unes avec les autres, même si parfois "IATR est perçue en certains milieux comme un concurrent on une "voleuse de projets" "(Bas-Saint-Laurent).

Les relations -actuelles et futures-avec les MRC présentent encore beaucoup de points

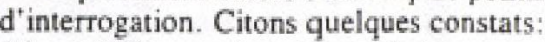
"Les MRC sont très jalouses de leur auto. nomie et de leur pouvoir régional" ' (BasSaint-Laurent). "Dans la connaissance des problemes et des solutions er dans la capacité de s'organiser se trowve la véritable capacité des ATR à participer au développement régional et à l'aménagement du territoire. Les MRC nous hiéconnaissent, nous craignent et se questionnent sur cette "nouvelle "force, ce nowveau pouvoir qui leur échappe et que constitue "'ATR' (Charle- 
voix). "Les MRC ne sont pas toujours a l'aise pour consulter les ATR. Certaines souhaiteraient avoir leur propre $A T R$ et auraient aimé se voir reconnaitre comme un région touristique, ce qui représente un grave danger d'une balkanisation touristique du Québec. Lorsqu'un projet amorcé (par whe ATR) fonctionthe bien, il arrive que des MRC s'en emparent et aient tendance a écaner l'ATR" (Estrie). "Les maires acceptent difficilement d'être *obligés* de se concenter et $n$ 'étaient surtout pas prêts. dans l'ensemble, à assumer les tâches d'un gouvemement régional. Les relarions entre notre ATR et les MRC ne sont vraiment pas claires. Nous intervenons, dans la mesure du possible, lors de consultations publiques et collaborons avec le ministere du Tourisme lors de la préparation des avis sectoriels des schémas d'aménagement. Norre röle est officiellement et strictement consultarif mème si, dans les faits, nous réussissons parfois à influencer le cours des décisions, afin que les schémas d'amènagement (dans le secteur du tourisme) soient plus concordanis avec la réalité de notre industrie et de ses composantes" (Gaspésie).

Mais si l'horizon est sombre, l'espoir n'a pas disparu: "La définition du rôle des $M R C$ ainsi que la précision de leur mandat sont un facteur qui risque à moyen terme de créer une confusion avec le róle des ATR puisque plusieurs MRC semblent vouloir elargir leur mandar bien au-delà de l'aménagement, Quoi qu'il en soit, une concertation entre les organismes regionaux et les instances politiques régionales (municipolités ou MRC) est essentielle au développement rationnel des ressources" (Pays-de-1'Érable). Cette concertation 'est donc le moyen de progresser harmonieusement; l'outil est le protocole d'entente et le Plan de développement dont I'ATR assure la maitrise d'oeuvre " (Charlevoix).

"Pour pouvoir travailler avec une certaine autonomie, une ATR ne doit pas se substituer au milieu mais l'appuyer " (Bas-SaintLaurent).

\section{Êtes-vous satisfait du décou- page actuel des régions touris- tiques?}

La plupart des ATR jugent satisfaisant le découpage des 18 régions touristiques québécoises et acceptent, parfois avec des nuances (Saguenay-Lac-Saint-JeanChibougamau, Coeur-du-Québec, BasSaint-Laurent, Gaspésie, Charlevoix, Montérégie), Jeurs limites territoriales actuelles.

L'Abitibi-Témiscamingue (rejointe en ce sens par Saguenay-Lac-Saint-JeanChibougamau) souhaite une révision de ce découpage: "On devrait viser la concordance entre régions administratives et régions touristiques. Soir qu' on augmente le nombre de régions administratives, soit qu'on diminue celui des régions touristiques ". Le Coeur-du-Québec emet une opi- nion plus nuancée: le découpage actuel peut peut-être satisfaire les intervenants du milieu mais ne rien signifier aux yeux des consommateurs qui continuent à associer Bas-Saint-Laurent et Gaspésie ou NouveauQuébec et Duplessis, par exemple.

Des ATR soulignent par ailleurs que ce découpage est le fruit d'une concertation à laquelle ont déjà participé toutes les régions du Québec. D'où l'opinion dominante: " $L$ e découpage actuel est satisfaisant parce que les ATR ont la souplesse et l'adaprabilité nécessaires pour contribue rà l'avancement de chaque partie du territoire" (Pays-del'Érable). "La composition d'une région doit reposer avant tout sur une homogénétié de ses composantes et respecter les sentiments d'appartenance des interverants". (Montérégie).

Le mandat actuel des ATR estil trop suffisamment ou pas assez économique? Devrait-il couvrir d'autres dimensions liées à la culture, au loisir, etc?

Une opinion résume toutes les autres: " $L$ e mandat des ATR doit être économique. S'il doit couvrir d'autres dimensions (comme celles de la culture ou du loisir), c'est dans le respect de l'autonomie de ces secteurs deja organisés" "(Saguenay-Lac-Saint-JeanChibougamau).

D'autres réponses viennent la compléter. l'enrichir: "Il serail faux de prétendre couvrir d'autres dimensions que celles liées au tourisme" "(Charlevoix) " L'énoncé ministériel nous semble satisfaisant: nous sommes avant tout des motivateurs, des promoteurs, des diffuseurs du produit touristique et non des gestionnaires d'equipements ou d'attractions" (Bas-SaintLaurent). "La culture, les loisirs et les aris sont tous des eléments importants pour ne pas dire essentiels à l'offre d'un produit touristique diversifié et bien structuré: ils s'intègrent très bien à l'intérieur d'un produit touristique car ils peuvent êre économiquement rentables' (Laurentides). $\mathrm{Ce}$ à quoi renchérit le Grand-Montréal: "La culture constitue un des principaux facteurs de promotion; les "événements majeurs* sont pour beaucoup des attraits touristiques".

Conclusion: "Les ATR ont à rendre touristiquement acceptables et exploitables des élements culturels et de loisir, etc. Pour ce faire, elles doivent collaborer avec les organismes sectoriels et les ministères" (Estrie). "En loisir, les responsabilités incombent aux municipalités, mais les ATR peuvent y jouer un rôle quand ces loisirs ont une certaine ampleur pouvant susciter l'intérềt des visiteurs" (Grand-Montréal).

Croyez-vous que les ATR -et la vôtre en particulier-devraient avoir des moyens supplémen- taires pour jouer un rôle plus actif dans le développement et I'aménagement régional?

Les réponses varient de "oui " à " absolument '"... Seule, l'ATR du Grand-Montréal s'avoue "très peut concemée" par cette question, à cause notamment de "l'importance de l'infrastructure touristique montréalaise" ". LATR de Lanaudière précise: "Les ATR devraient avoir plus de movens dans la mesure de l"implication financiere de leurs milieur respectifs. Celte formule, tout en étant contraignante, est la seule qui garantit le représentation des intérêts du milieu" (Lanaudière).

Le désir de moyens supplémentaires s'explique par le besoin de ressources financieres et humaines qui s'avéteraient aujourd hui insuffisantes. L'embauche en chaque région d'un agent de développement sur une base permanente se présente, entre autres, comme un moyen indispensable, ne serait-ce que pour assurer le suivi du Plan de développement. "Ce plan exigera un suivi effectif sur le terrain, tant dans la mise a jour es l'adéquation des schémas que dans la réalisation des projets de développement" (Charlevoix)

Citons deux témoignages. "Nous $n$ 'avons présentement aucune ressource tant financière qu humaine pour répondre aux attentes des multiples intervenants dans l'aménagement et le développement de notre territoire. N'oubions pas que nous sommes l'une des rares structures orgamisées à partir du milieu qui touche, par son intervention, à presque tous les secteurs de notre économie. Nous sommes en quelque sorte des specialistes et des experts de la polyvalence. Avec deux permanents (une directrice générale essouflée et uné secrétaire non moins essouffée), comment peuton sérieusement et adequatement servir notre région?" (Gaspésie).

"Pour pouvoir agir et intervenir, les ATR comme la nôrre, qui ne peuvent comprer que sur whe foule de petits intervenants dotés de moyens très restreints, sont continuellement a la recherche de programmes et de sources de financement pour garantir leur survie. Ces programmes de création d'emplois sont des palliarifs qui rendent la crédibilité difficile d̀ s'installer et qui ne constituent pas un moyen efficace d'intervention. Notre ATR, avec un budget de fonctionnement raisonnable et le personnel requis, pourrait s occuper davantage de tourisme et awoir une action plus profitable dans le milieu" "(Bas-Saint-Laurent).

Et celle-ci de conclure sur un ultime espoir: "C'est ce qui semble se dessiner à l'horizon avec une nouvelle proposition de financement de la part du ministère". Mais. avec la nouvelle vague de coupures et de restrictions budgétaires, en sera-t-il vraiment ainsi? $f$ 